\title{
Florid osseous dysplasia with superimposed mandibular osteomyelitis
}

\author{
Sayed Ali • Sunita Nankoo • Ali B. Syed • Jie Yang
}

Published online: 19 November 2013

(C) ISS 2013

$\mathrm{CT}$ images show four-quadrant (bilateral mandibular and maxillary) exuberant sclerosis. Sclerosis surrounds several teeth including the premolar and molar teeth. There is mild expansile bone remodeling but no cortical thickening, periosteal reaction, or soft tissue inflammatory changes. There are associated destructive osteolytic changes within the mandible, however no corresponding osteolysis is seen in the maxilla.

The pronounced sclerosis and expansile remodeling, with areas of osteolysis in the mandible, raises the diagnostic possibility of a chronic sclerosing bone dysplasia, or infection. Paget's disease can involve the maxilla or mandible and is often polyostotic with bone enlargement, sclerosis, trabecular coarsening, and cortical thickening during the osteoblastic phase [1]. However, this case demonstrates no cortical thickening or trabecular coarsening, and the maxillary lesions also have a thin surrounding radiolucent rim, discounting Paget's disease as a possibility.

Bilateral maxillary and mandibular involvement with exuberant sclerosis and mild expansile remodeling of bone is consistent with florid osseous dysplasia (FOD). Fibroosseous lesions are benign entities that possess both fibrous and osseous components, and include reactive, neoplastic, and dysplastic processes [2-4]. These entities include fibrous dysplasia, focal osseous dysplasia, and florid osseous dysplasia (FOD). Fibrous dysplasia does not have a periodontal ligament origin unlike the other two entities, and its radiographic appearance ranges from an osteolytic lesion to a sclerotic or "ground glass" appearance with a loss of corticomedullary differentiation, unlike focal osseous dysplasia and FOD where the corticomedullary differentiation is typically preserved. Focal osseous dysplasia is similar to FOD in its origin from the periodontal ligament and its radiographic appearance,

The case presentation can be found at doi: 10.1007/s00256-013-1763-8

S. Ali $(\bowtie) \cdot$ S. Nankoo $\cdot$ A. B. Syed $\cdot$ J. Yang Department of Radiology, Temple University Hospital, 3401 North Broad Street, Philadelphia, PA 19140, USA e-mail: saychink@gmail.com however FOD is more extensive, involving multiple quadrants of the facial bones, whereas focal osseous dysplasia is solitary.

While the sclerosis and remodeling is well explained by FOD, this does not explain the destructive changes in the mandible. Chronic sclerosing osteomyelitis can present with pain and swelling about tooth-bearing areas and typically occurs in the mandible, with radiographic changes of periosteal reaction, bone expansion, and sclerosis [5]. One would reasonably expect significant soft tissue swelling or periosteal reaction in some or all of the lesions if chronic sclerosing osteomyelitis is the primary etiology, and although minimal anterior mandibular soft tissue inflammatory changes are present, there is no significant swelling or periosteal reaction. In particular, the presence of multiquadrant disease makes this entity very unlikely. However, the areas of osteolysis in the mandible demonstrate sequestrae, which are not present in the maxillae. Therefore, despite the absence of significant soft tissue swelling or periosteal reaction, the mandibular changes are consistent with suppurative osteomyelitis secondary to FOD.

In FOD, biopsy, if performed, reveals dysplastic trabeculae intermixed with fibrous stroma and with incomplete stromal vasculature [6]. FOD typically involves the jaw bones and is characterized by replacement of normal bone by dysplastic connective tissue and abnormal bone, and is believed to arise from the periodontal ligament [7]. FOD typically demonstrates extensive multifocal bilateral involvement of the mandibular molar/premolar region, and is usually symmetric [8]. There is often concomitant maxillary involvement, as in this case. Radiographic findings include confluent and mildly expansile or nonexpansile sclerotic lesions with a circumferential surrounding thin radiolucency [9], although the lesions are initially radiographically radiolucent with progressive sclerosis. Occasionally, a simple bone cyst may be associated with FOD, and this may result in more expansion or fenestration of the cortices of the maxilla and/or mandible. Patients with FOD are typically more than 45 years, and there is a predilection for black females. Caucasians and Asians are less commonly 
affected [9]. Radiography is sufficient for diagnosis of asymptomatic patients, and biopsy is usually not indicated. Complaints of pain are usually the result of superimposed infection. Osteomyelitis may ensue following trauma, due to the avascular nature of FOD [10]. Secondary infection is a dilemma, as the administration of antibiotics alone may not be successful [11], and the infected areas may require surgical debridement.

In summary, exuberant bilateral mandibular and maxillary osteosclerosis should raise the concern for florid osseous dysplasia, a rare benign entity that does not require biopsy. However, the presence of concomitant infection should be excluded, as it is a relatively common complication that is unfortunately difficult to treat.

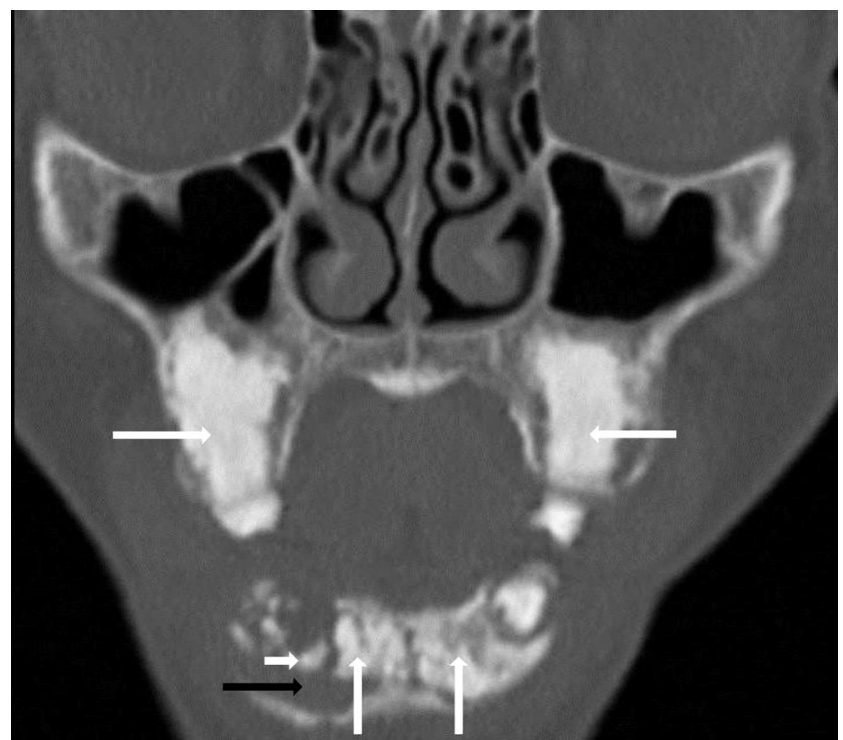

Coronal reformatted CT showing four-quadrant osteosclerosis (white arrows), and osteolytic infected bone in the mandible (black arrow) with sequestrum formation (short white arrow)
Conflict of interest The authors declare that they have no conflicts of interest

\section{References}

1. Karunakaran K, Murugesan P, Rajeshwar G, Babu S. Paget's disease of the mandible. J Oral Maxillofac Pathol JOMFP. 2012;16: $107-9$.

2. Eversole R, Su L, ElMofty S. Benign fibro-osseous lesions of the craniofacial complex a review. Head Neck Pathol. 2008;2: 177-202.

3. Chi A. Fibro-osseous lesions of the jaws. In: Neville B, Damm D, Allen M, Bouquot J, editors. Oral Maxillofac. Pathol. Elsevier Saunders; 2009. p. 635-48.

4. Potochny EM, Huber AR. Focal osseous dysplasia. Head Neck Pathol. 2011;5:265-7.

5. Orpe EC, Lee L, Pharoah MJ. A radiological analysis of chronic sclerosing osteomyelitis of the mandible. Dento Maxillo Facial Radiol. 1996;25:125-9.

6. Drazić R, Minić AJ. Focal cemento-osseous dysplasia in the maxilla mimicking periapical granuloma. Oral Surg Oral Med Oral Pathol Oral Radiol Endod. 1999;88:87-9.

7. Kim J-H, Song B-C, Kim S-H, Park Y-S. Clinical, radiographic, and histological findings of florid cemento-osseous dysplasia: a case report. Imaging Sci Dent. 2011;41:139-42.

8. Barnes L, Eveson JW, Reichart P, Sidransky D, editors. Pathology and genetics of head and neck tumours. Lyon: IARC Press; 2005.

9. Miyake M, Nagahata S. Florid cemento-osseous dysplasia report of a case. Int J Oral Maxillofac Surg. 1999;28:56-7.

10. Waldron CA. Fibro-osseous lesions of the jaws. J Oral Maxillofac Surg Off J Am Assoc Oral Maxillofac Surg. 1993;51: 828-35.

11. $\mathrm{Su}$ L, Weathers DR, Waldron CA. Distinguishing features of focal cemento-osseous dysplasia and cemento-ossifying fibromas. II. A clinical and radiologic spectrum of 316 cases. Oral Surg Oral Med Oral Pathol Oral Radiol Endod. 1997;84:5409. 